\title{
Comparison of radiation dose spillage from the Gamma Knife Perfexion with that from volumetric modulated arc radiosurgery during treatment of multiple brain metastases in a single fraction
}

\author{
Clinical article
}

\author{
Daniel McDonald, M.S., ${ }^{1}$ John Schuler, M.D., ${ }^{1}$ Istvan Takacs, M.D., ${ }^{2}$ Jean Peng, Ph.D., ${ }^{1}$ \\ Joseph Jenrette, M.D., ${ }^{1}$ And Kenneth VAnek, Ph.D. ${ }^{1}$
}

\author{
Departments of ${ }^{1}$ Radiation Oncology and ${ }^{2}$ Neurosurgery, Medical University of South Carolina, Charleston, \\ South Carolina
}

\begin{abstract}
Object. The objective of this study was to examine radiation dose distributions created by 2 competing radiosurgery modalities for treating multiple brain metastases: single-isocenter volumetric modulated arc radiosurgery (VMAS) and Gamma Knife Perfexion (GKP). In addition, the effectiveness of multiple radiosurgery quality metrics was evaluated and compared between these advanced treatment modalities.

Methods. Seven anonymized MRI data sets, each showing 2-5 metastases, were used to create plans on each system. The GammaPlan (version 10.1) program was used for planning of GKP. A neurosurgeon contoured the volumes to be treated, and no planning target volume expansion was used. A prescription dose coverage of $\geq 99 \%$ was achieved for each tumor volume. The Philips Pinnacle (version 9.2) program was used for planning of VMAS, using the SmartArc optimization algorithm for delivery on a Varian iX linear accelerator. Contours were transferred from GammaPlan, and again no planning target volume expansion was used. Between 2 and 5 arcs with table angles of $90^{\circ}-270^{\circ}$ were used. Again, a V100\% of $\geq 99 \%$ was achieved for each tumor volume. After planning, the MRI scans, tumor volumes, and dose information from each plan were exported according to the Digital Imaging and Communications in Medicine standard to the VelocityAI program for analysis. Brain dose-volume histograms (DVHs) for normal brain tissues were generated, and the volume of these tissues receiving $20 \%-90 \%$ of the prescription dose was tabulated. Finally, the prescription isodose to tumor volume ratio (PITV; Shaw et al., 1993), conformity index (CI; Paddick, 2000), gradient index (GI, Paddick and Lippitz, 2006), and conformity/gradient index (CGI, Wagner et al. 2003) were calculated for each plan. Both the PITV and CI have ideal values of 1, while the GI and CGI have ideal values of lowest and highest achievable, respectively.

Results. The DVHs consistently showed that with VMAS a higher amount of normal brain tissues received each dose level than with GKP. These increases were largest for lower isodose levels, with the volumes of normal brain that received $20 \%-50 \%$ and $60 \%-90 \%$ of the prescription dose showing average increases of $403 \%$ and $227 \%$, respectively. Prescription isodose conformality showed only minor differences between the 2 modalities. Radiosurgery quality metrics including measures of the dose gradient (GI and CGI) indicated that the GKP plan was superior in each case, with respective average GI and CGI values of 3.04 and 57.75 for GKP and of 10.22 and 10.85 for VMAS. Metrics evaluating prescription isodose conformality alone differed only slightly between the modalities. Average respective PITV and CI values were 2.13 and 0.53 for GKP and 2.27 and 0.51 for VMAS.

Conclusions. Stereotactic radiosurgery plans for the treatment of multiple metastases with VMAS delivered significantly more dose to the normal brain tissues than plans for GKP. Radiosurgery quality metrics including a measure of the dose gradient are better suited to providing contrast between modern radiosurgery treatment platforms.
\end{abstract}

(http://thejns.org/doi/abs/10.3171/2014.7.GKS141358)

$\begin{aligned} & \text { KEY WoRds } \\ & \text { volumetric modulated arc radiosurgery } \\ & \text { conformality }\end{aligned}$

$\mathrm{H}$

ISTORICALLY, the appearance of multiple brain metastases indicated a dismal prognosis, and options for simultaneous treatment of these metas-

\footnotetext{
Abbreviations used in this paper: CGI = conformity/gradient index; CI = conformity index; DVH = dose-volume histogram; GI = gradient index; GK4C = Gamma Knife model 4C; GKP = Gamma Knife Perfexion; MLC = multileaf collimator; PITV = prescription isodose to tumor volume ratio; VMAS = volumetric modulated arc radiosurgery; WBRT $=$ whole-brain radiotherapy.
}

tases were limited. Resection has been shown to improve quality of life and increase overall survival for patients with a single, accessible symptomatic lesion. ${ }^{17}$ In addition, removal of $2-3$ metastases exerting a mass effect has proven appropriate because the benefits of the resulting decompression are immediate. Although Bindal et al. ${ }^{2}$ found resection of multiple lesions to be beneficial, none of the patients they treated had more than 4 metastases removed and none had more than 3 craniotomies. In current clinical practice, the extent of surgical intervention 
must be dictated by specific considerations for each individual patient. The American Association of Neurological Surgeons' treatment guidelines, ${ }^{8}$ the World Federation of Neurosurgical Societies review, ${ }^{24}$ and comprehensive overviews by $\mathrm{Suh}^{20}$ and Sawaya and Wildrick ${ }^{18}$ all offer useful reviews.

Radiation therapy also has an established role for controlling multifocal brain disease. Whole-brain radiotherapy (WBRT) is effective, especially for treating radiosensitive cancers..$^{10}$ However, the radiation dose delivered to the normal brain tissues and to radiosensitive organs, such as the optic nerves, limits its use. Studies have shown that the irradiation of normal brain tissues may have undesirable neurocognitive effects, including memory loss. ${ }^{3,23}$ As a result of an increased recognition of the limitations of WBRT, stereotactic radiosurgery, which allows for precise targeting of brain tumors and spares normal brain tissues, has grown in popularity. While previous methods of radiosurgical treatment, such as linear accelerator-based cone or conformal beam; $\mathrm{Cy}$ berKnife (Accuray); and Gamma Knife models U, B, C, and $4 \mathrm{C}$ (Elekta), did allow for the treatment of multiple metastases, treatment of a large number of targets was prohibitively time consuming. For this reason, radiosurgery was typically reserved for patients with few tumors.

Two recently developed radiosurgery modalities offer treatment of a large number of metastases in a single fraction. Gamma Knife Perfexion (GKP) (Elekta) is the most recent version of the Gamma Knife treatment platform. Automatic collimator adjustment and table positioning allow for smooth sequential treatment of brain metastases. ${ }^{20}$ In addition, the GammaPlan treatment planning system allows the user to quickly and automatically mark previously treated tumors, reducing the complexity of treating new metastases with additional treatment sessions if necessary. Single-isocenter volumetric modulated arc radiosurgery (VMAS) is an arc-based treatment delivered with a traditional linear accelerator. During delivery, the gantry rotates around a single isocenter placed in the center of a group of metastases. Dynamic multileaf collimation shapes the dose distribution, enabling the simultaneous treatment of an entire group of tumors. ${ }^{4}$ The use of a single isocenter rather than of separate isocenters for each metastasis greatly reduces setup time, making the treatment of larger numbers of metastases feasible.

These radiosurgery techniques have changed the way in which patients with multiple metastases are treated. Given that metastatic brain disease, by definition, represents Stage 4 cancer, its overall prognosis remains grave. However, in many patients, intracranial disease can be successfully managed with radiosurgery to treat any initial metastases, followed by surveillance imaging and additional radiosurgical treatments should any new metastases be detected during follow-up.

This course of treatment strongly relies on exceedingly conformal dose distributions. Studies have shown that VMAS and GKP may produce similar results when evaluated with traditional radiosurgery metrics. ${ }^{21}$ These metrics typically place importance on conformality of the prescription isodose line and coverage of the target. ${ }^{15,19}$ However, as discussed above, the ability to treat multiple cranial metastases with radiosurgery, as well as improved extracranial disease control with novel chemotherapeutic, immunotherapeutic, and biological agents, has greatly increased the total number of metastases that may be treated in a single patient. As a result, different measures of plan quality may be required to evaluate the equivalence of VMAS and GKP. In particular, spillage of low radiation doses to normal brain tissues is of concern. As the number of tumors treated and the number of treatment sessions increase, these low doses accumulate. Such dose accumulation may eventually prohibit additional treatments because of the lower dose tolerances of sensitive cranial structures, or it may cause the long-term cognitive deficits associated with WBRT.

Previous research has studied this low-dose radiation spillage by comparing VMAS, GKP, and other radiosurgery treatment platforms on a single-patient data set using the Varian RapidArc delivery system with 2.5-mm micro multileaf collimators (MLCs) (Varian). ${ }^{11,12,14}$ This work showed that the VMAS plans resulted in higher dose spillage and that lower isodose lines showed the greatest increase in spillage. A previous study of patients with multiple metastases has also compared an older version of the Gamma Knife, model 4C, to VMAS, also using the RapidArc and micro MLCs. ${ }^{21}$ This work showed significantly improved prescription isodose conformality for the VMAS plans. Differences in dose spillage to healthy brain tissues were not found to be statistically significant, but average values were found to be improved for the Gamma Knife.

The work presented here sought to add to the work discussed above by expanding the study to multiple patient data sets planned with the more modern GKP. Also novel was the use of the Philips Pinnacle VMAS algorithm SmartArc (Philips) optimization algorithm to generate plans for delivery on a Varian iX with Millenium MLCs (5-mm width in the central $20 \mathrm{~cm}$ ). Finally, multiple radiosurgery quality metrics were calculated for each plan in an effort to determine which metrics may be most useful for evaluating plans created for these advanced treatment modalities.

\section{Methods}

\section{Image Set Selection}

Seven anonymized patient image sets of T1-weighted MRI scans with a slice thickness of $1 \mathrm{~mm}$ were selected for planning and analysis. Patients had been given gadolinium contrast medium prior to imaging. Because these patients were treated with the GKP, the appropriate Leksell MRI localizer was used during imaging. Two image sets showed 2 metastases, 2 showed 3 metastases, 1 showed 4 metastases, and 2 showed 5 metastases (Table 1). These image sets were randomly chosen on the basis of the number of metastases alone to provide a wide selection of different potential clinical situations. The size and position of each metastasis were not considered during image set selection. Total cumulative tumor volumes ranged from 1.56 to $41.7 \mathrm{~cm}^{3}$. While we have treated up to 15 metastases in 1 treatment session in our clinic, a 


\section{Gamma Knife Perfexion versus VMAS}

TABLE 1: General image set parameters*

\begin{tabular}{cccc}
\hline Image Set & No. of Targets & $\begin{array}{c}\text { Total Tumor Vol } \\
\left(\mathrm{cm}^{3}\right)\end{array}$ & $\begin{array}{c}\text { Prescription } \\
\text { Dose (Gy) }\end{array}$ \\
\hline 1 & 2 & 3.71 & 15.0 \\
2 & 2 & 6.57 & 15.0 \\
3 & 3 & 21.28 & 14.0 \\
4 & 3 & 9.29 & 14.0 \\
5 & 4 & 41.70 & 14.0 \\
6 & 5 & 1.63 & 13.0 \\
7 & 5 & 1.56 & 12.5 \\
\hline
\end{tabular}

* Each tumor in a given image set received the reported prescription dose.

group of patients with more common numbers of metastases was chosen for this study to provide results more applicable to most treatment centers. To simplify and standardize the analysis, prescription doses were the same for all metastases in a given image set.

\section{Planning for the GKP}

Gamma Knife Perfexion planning was performed using the GammaPlan software program (version 10.1). The MRI data sets were imported and tumors localized according to a standard procedure. Skull measurements were entered to generate an external contour for the calculation of beam attenuation. No heterogeneity corrections were used. A neurosurgeon contoured the volumes to be treated and placed targets around these volumes. No planning target volume expansion was used. Shots were placed and adjusted manually by a medical physicist with 5 years' experience planning both accelerator-based and Gamma Knife radiosurgery. No limit was placed on the number of shots or the allowed collimator combinations. A V100\% of $\geq 99 \%$ was achieved for each tumor volume in each plan, and all targets were prescribed to the $50 \%$ isodose line. The total number of shots used per plan ranged from 17 to 59 (Table 2). All 3 collimator sizes (4, 8 , and $16 \mathrm{~mm}$ ) were used on each plan. A gamma angle of $90^{\circ}$ was used whenever possible, except when prohibited by a potential frame collision. Beam-on times ranged from 56.1 to 134.2 minutes.

\section{TABLE 2: Planning parameters for GKP*}

\begin{tabular}{ccccc}
\hline Image Set & $\begin{array}{c}\text { No. of } \\
\text { Targets }\end{array}$ & $\begin{array}{c}\text { No. of Total } \\
\text { Shots }\end{array}$ & $\begin{array}{c}\text { Gamma Angles } \\
\text { Used }\left(^{\circ}\right)\end{array}$ & $\begin{array}{c}\text { Beam-On } \\
\text { Time (mins) }\end{array}$ \\
\hline 1 & 2 & 19 & 90 & 56.1 \\
2 & 2 & 22 & $90 \& 110$ & 57.3 \\
3 & 3 & 48 & 90 & 127.8 \\
4 & 3 & 33 & 90 & 112.4 \\
5 & 4 & 59 & 90 & 134.2 \\
6 & 5 & 17 & 90 & 57.4 \\
7 & 5 & 34 & $70 \& 90$ & 84.6 \\
\hline
\end{tabular}

* A prescription isodose line of $50 \%$ and collimator sizes of 4,8 , and 16 $\mathrm{mm}$ were used in all image sets.

\section{Planning for Pinnacle SmartArc VMAS}

SmartArc VMAS plans were created using the Philips Pinnacle software program (version 9.2). The MRI data sets and tumor contours were exported from GammaPlan and imported into the Pinnacle planning system. In addition, the measurement-generated external skull contour was also transferred and set to a density of $1 \mathrm{~g} /$ $\mathrm{cm}^{3}$. This allowed for homogeneous planning with the Pinnacle system, without the need for a CT data set. As in GammaPlan, no planning target volume expansion was used. The VMAS planning was carried out by a medical physicist with 5 years' experience planning both accelerator-based and Gamma Knife radiosurgery. Arc placement and table angle varied for each case; 2-5 arcs were used, with table angles ranging from $90^{\circ}$ to $270^{\circ}$. Arc placement and table angles were selected to achieve the best possible conformality, while taking into consideration realistic clinical constraints.

Excellent work has previously examined the optimum planning parameters for single-isocenter VMAS., 1,9 We referred to these studies while constructing the VMAS plans presented in this paper. Objectives included optimizing minimum dose and dose-volume constraints on the tumor volumes, maximum dose and dose-volume constraints on various dose rings, and maximum equivalent uniform dose constraints for normal brain tissues. Appropriate dose constraints were placed on any relevant organs at risk, such as the brainstem, optic structures, or skin. When necessary, additional dose-shaping structures were also contoured and used. Control point spacing was $4^{\circ}$ for each plan (Table 3 ). The average arc length was $\geq$ $210^{\circ}$. The total number of monitor units per plan ranged from 1898 to 3735 . As with the GKP, a V100\% of $\geq 99 \%$ was achieved for each tumor volume in each plan.

\section{Plan Analysis}

For analysis, the MRI data set, tumor contours, and Digital Imaging and Communications in Medicine dose information for each plan were exported to a third-party software program, VelocityAI (Velocity Medical Solutions). This ensured equivalent analysis and eliminated possible bias created by differences in the evaluation algorithms within each planning system. In VelocityAI, a normal brain contour was generated on each data set by contouring the brain and subtracting the tumor volumes. Dose-volume histograms (DVHs) for normal brain tissues were generated for each plan, and the amount of normal brain tissue receiving 20\%, 30\%, 40\%, 50\%, 60\%, 70\%, $80 \%$, or $90 \%$ of the prescription dose was calculated for each plan. In addition, isodose line overlays were created, allowing for the simultaneous graphical examination of the dose spillage in each modality. Finally, a number of different radiosurgery plan quality metrics were calculated for each plan and compared. Table 4 outlines the parameters of each quality metric.

The prescription isodose to tumor volume ratio (PITV), proposed by Shaw et al., ${ }^{19}$ compares the volume of the prescription isodose to the tumor volume. The conformity index (CI), proposed by Paddick, ${ }^{15}$ also examines the volume of the prescription isodose in comparison 
TABLE 3: Planning parameters for VMAS*

\begin{tabular}{ccccccc}
\hline Image Set & $\begin{array}{c}\text { No. of } \\
\text { Targets }\end{array}$ & $\begin{array}{c}\text { Prescription Isodose } \\
\text { Line (\%) }\end{array}$ & No. of Arcs & $\begin{array}{c}\text { Average Arc } \\
\text { Length }\left(^{\circ}\right)\end{array}$ & Table Angles $\left(^{\circ}\right)$ & $\begin{array}{c}\text { No. of Monitor } \\
\text { Units }\end{array}$ \\
\hline 1 & 2 & 97.0 & 4 & 270 & $105,170,190,255$ & 2631 \\
2 & 2 & 93.7 & 4 & 220 & $90,145,180,225$ & 1898 \\
3 & 3 & 92.0 & 2 & 360 & 175,185 & 2592 \\
4 & 3 & 92.0 & 4 & 212 & $90,110,180,250$ & 2760 \\
5 & 4 & 76.6 & 5 & 218 & $110,170,190,250,270$ & 3735 \\
6 & 5 & 94.9 & 4 & 210 & $90,110,180,250$ & 2961 \\
7 & 5 & 94.5 & 4 & 360 & 170,190 & 3031 \\
\hline
\end{tabular}

* A control point-spacing angle of $4^{\circ}$ was used in all image sets.

with the tumor volume, but adds a measure of tumor coverage. These metrics are concerned primarily with the prescription isodose. The gradient index (GI), proposed by Paddick and Lippitz ${ }^{16}$ compares the volume of the isodose for $50 \%$ of the prescription dose with the volume of the prescription isodose. Finally, the conformity/gradient index (CGI), proposed by Wagner et al.,22 includes parameters evaluating both conformality of the prescription isodose, as well as a gradient term evaluating dose falloff. Both the GI and CGI consider, at least in part, the volume of isodose lines less than the prescription dose when determining plan quality. For a detailed explanation of each metric, and instructions for calculation, the reader is directed to the appropriate references listed above.

\section{Results}

\section{Normal Brain DVH and Graphical Comparison}

Dose-volume histograms of the normal brain contour, calculated for each plan, consistently showed that compared with GKP, VMAS increased the amount of normal brain tissues receiving each radiation dose level examined. Figure 1 shows a normal brain DVH curve comparison for Image Set 6. Similar curve comparisons were generated for each image set. Table 5 shows the volumes of normal brain tissues receiving $20 \%, 30 \%, 40 \%, 50 \%, 60 \%, 70 \%, 80 \%$, or $90 \%$ of the prescription dose for each plan. Table 6 gives the percentage increase, from GKP to VMAS, in normal brain receiving a given percentage of the prescription dose for each image set. The volume of the prescription isodose is also provided for reference.

Graphical comparisons were also conducted by over- laying selected isodose lines from both GKP and VMAS. Figure 2 shows a sampling of the isodose line overlays generated for each image set. To better visualize differences in conformality and dose spillage across multiple dose levels, Fig. 2A shows only the prescription isodose (14 Gy) from each modality. Figure 2B shows the prescription and 7-Gy isodose lines, Fig. 2C the prescription and 4-Gy isodose lines, and Fig. 2D-F the same isodose levels for a second data set.

\section{Comparison of Radiosurgery Plan Quality Metrics}

Table 7 shows the quality metrics calculated for each plan. Both PITV and CI showed only a minor dependence on treatment modality. Gamma Knife Perfexion had average PITV and CI values of 2.13 and 0.53 , respectively, while VMAS had average PITV and CI values of 2.27 and 0.51, respectively. Both GI and CGI showed significant dependence on treatment modality. Gamma Knife Perfexion had average GI and CGI values of 3.04 and 57.75, respectively, while VMAS had average GI and CGI values of 10.22 and 10.85 , respectively.

\section{Discussion}

\section{Normal Brain DVH and Graphical Comparison}

The DVHs of normal brain tissues consistently showed that these tissues received a higher radiation dose from VMAS than from GKP. These increases were most significant at lower isodose levels, with V20\%-V50\% showing an average increase of $403 \%$, and $\mathrm{V} 60 \%-\mathrm{V} 90 \%$ showing an average increase of $227 \%$. Prescription iso-

TABLE 4: Parameters of the quality metrics used in the radiosurgery planning*

\begin{tabular}{|c|c|c|c|}
\hline Metric & Formula & Ideal Value & Reference \\
\hline PITV & PIV/TV & 1 & Shaw et al., 1993 \\
\hline $\mathrm{Cl}$ & $\left(\mathrm{TV}_{\mathrm{PIV}}\right)^{2} /(\mathrm{TV} \times \mathrm{PIV})$ & 1 & Paddick, 2000 \\
\hline GI & $\mathrm{PIV}_{50 \%} / \mathrm{PIV}$ & lowest achievable & Paddick \& Lippitz, 2006 \\
\hline CGI & $\left\{100(\right.$ TV/PIV $\left.)+100-\left[100\left(R_{E f f, 50 \% R x}-R_{E f f, R x}\right)-0.3_{c m}\right]\right\} / 2$ & highest achievable & Wagner et al., 2003 \\
\hline
\end{tabular}




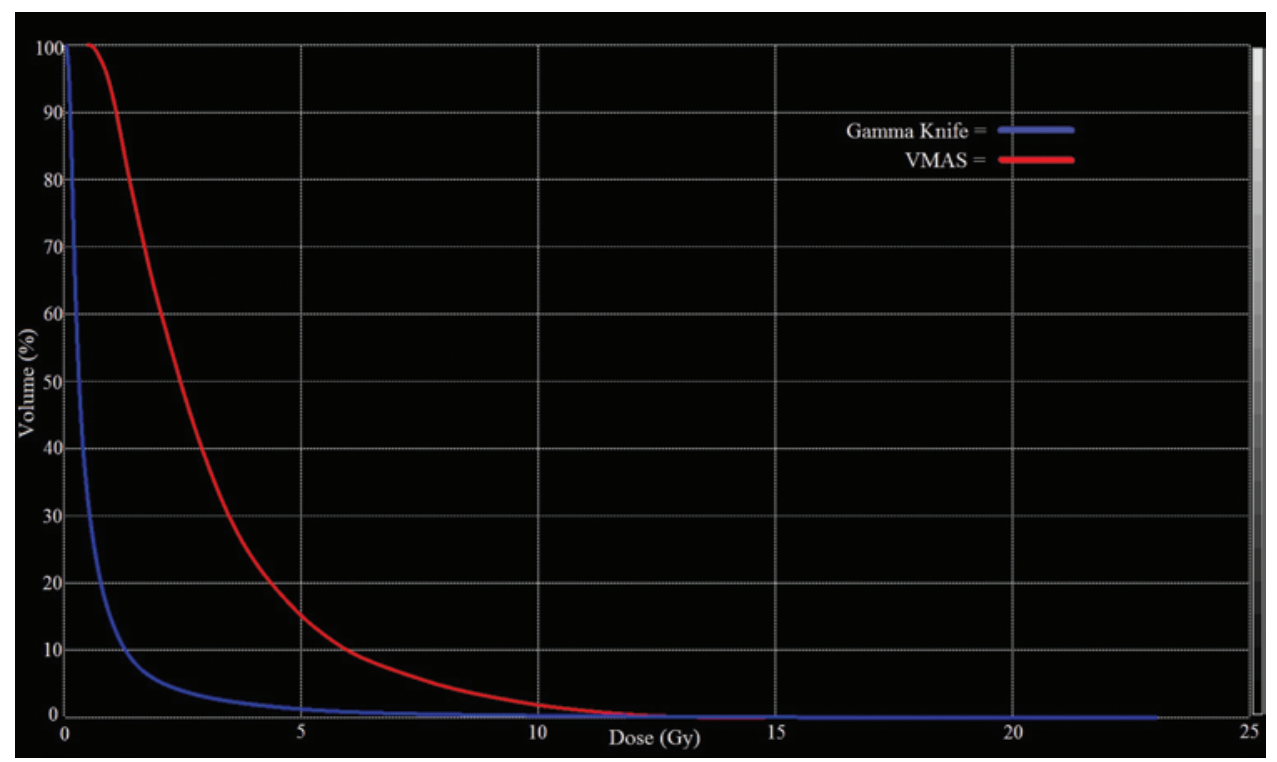

FIG. 1. Normal brain DVH overlay with GKP shown in blue and VMAS in red. The DVHs were calculated using VelocityAI. In all cases, compared with VMAS, the GKP improved the DVH for the normal brain.

dose volumes showed only small differences between GKP and VMAS. This indicates that, while VMAS is capable of producing radiosurgery plans with prescription isodose conformalities similar to GKP, the GKP treatment modality produces superior conformality at the lower isodose levels.

An examination of each treatment platform may provide insight into these differences. Gamma Knife Perfexion uses 192 individual cobalt-60 sources to deliver radiation to a focused isocenter. As each shot is completed, the sources are blocked, and the treatment couch is shifted to reposition the radiation isocenter, effectively shifting all 192 cobalt-60 beam paths. This procedure results in virtually no transit dose, that is, a dose delivered inadvertently as the table shifts position. In addition, by shifting the treatment isocenter for each shot, beam overlap in most of the normal brain tissues is kept to a minimum, producing highly conformal dose distributions across all dose levels.

Single-isocenter VMAS uses a single-isocenter point for all treatment arcs. During each treatment arc, the MLCs adjust dynamically, attempting to deliver dose to as many tumor volumes as possible. Dose is delivered continuously, and, as a result, dose spillage is delivered to the normal brain as the MLCs move to cover different tumor volumes. Increasing numbers of metastases

TABLE 5: Normal brain dose statistics reported by absolute volume

\begin{tabular}{|c|c|c|c|c|c|c|c|c|}
\hline \multirow[b]{2}{*}{ Rx Dose } & \multirow[b]{2}{*}{ Modality } & \multicolumn{7}{|c|}{ Vol of Healthy Brain Ordered by Image Set $\left(\mathrm{cm}^{3}\right)$} \\
\hline & & 1 & 2 & 3 & 4 & 5 & 6 & 7 \\
\hline \multirow[t]{2}{*}{$\mathrm{V} 20 \%$} & GKP & 68.9 & 82.2 & 228.3 & 80.2 & 355.2 & 54.0 & 43.4 \\
\hline & VMAS & 186.0 & 270.0 & 884.0 & 416.8 & 1087.0 & 667.3 & 342.3 \\
\hline \multirow[t]{2}{*}{ V30\% } & GKP & 34.6 & 44.7 & 115.3 & 46.2 & 204.7 & 28.6 & 22.9 \\
\hline & VMAS & 88.1 & 125.7 & 517.7 & 227.0 & 641.7 & 357.0 & 154.1 \\
\hline \multirow[t]{2}{*}{ V $40 \%$} & GKP & 21.2 & 28.8 & 70.0 & 31.2 & 132.7 & 17.2 & 13.7 \\
\hline & VMAS & 51.7 & 76.4 & 295.7 & 120.7 & 363.8 & 205.1 & 89.7 \\
\hline \multirow[t]{2}{*}{ V $50 \%$} & GKP & 14.7 & 20.2 & 46.9 & 22.1 & 89.8 & 11.0 & 9.4 \\
\hline & VMAS & 33.7 & 50.8 & 161.9 & 69.6 & 205.0 & 121.5 & 57.7 \\
\hline \multirow[t]{2}{*}{ V $60 \%$} & GKP & 10.6 & 14.7 & 32.8 & 16.4 & 61.1 & 7.5 & 6.8 \\
\hline & VMAS & 23.2 & 36.1 & 101.2 & 48.3 & 116.0 & 78.4 & 37.6 \\
\hline \multirow[t]{2}{*}{ V70\% } & GKP & 7.6 & 10.5 & 23.3 & 12.2 & 41.9 & 5.7 & 5.1 \\
\hline & VMAS & 16.1 & 26.3 & 68.0 & 33.9 & 69.5 & 46.6 & 25.6 \\
\hline \multirow[t]{2}{*}{ V $80 \%$} & GKP & 5.6 & 7.3 & 16.3 & 9.0 & 28.1 & 4.4 & 3.8 \\
\hline & VMAS & 10.8 & 18.8 & 45.3 & 24.0 & 43.3 & 25.1 & 15.6 \\
\hline \multirow[t]{2}{*}{ V90\% } & GKP & 4.0 & 4.9 & 10.8 & 6.4 & 17.4 & 3.4 & 2.8 \\
\hline & VMAS & 6.6 & 12.0 & 26.4 & 15.2 & 24.3 & 11.6 & 7.8 \\
\hline
\end{tabular}


D. McDonald et al.

TABLE 6: Healthy brain dose statistics reported as percentage increase from GKP to VMAS*

\begin{tabular}{|c|c|c|c|c|c|c|c|c|c|c|}
\hline \multirow{2}{*}{$\begin{array}{c}\text { Image } \\
\text { Set }\end{array}$} & \multicolumn{2}{|c|}{ Rx Isodose Line $\mathrm{Vol}\left(\mathrm{cm}^{3}\right)$} & \multicolumn{8}{|c|}{ Percentage Increase $(\%)=\left[\left(\mathrm{V}_{\mathrm{NB}, \mathrm{VMAS}}-\mathrm{V}_{\mathrm{NB}, \mathrm{GKP}}\right) / \mathrm{V}_{\mathrm{NB}, \mathrm{GKP}}\right] \times 100$} \\
\hline & GKP & VMAS & V20\% & V30\% & V $40 \%$ & V50\% & V60\% & V70\% & V80\% & V90\% \\
\hline 1 & 6.5 & 6.6 & 170 & 155 & 144 & 129 & 119 & 112 & 93 & 65 \\
\hline 2 & 9.5 & 9.9 & 228 & 181 & 165 & 151 & 146 & 150 & 158 & 145 \\
\hline 3 & 33.3 & 35.5 & 287 & 349 & 322 & 245 & 209 & 192 & 178 & 144 \\
\hline 4 & 18.1 & 18.2 & 420 & 391 & 287 & 215 & 195 & 178 & 167 & 138 \\
\hline 5 & 56.3 & 58.7 & 206 & 213 & 174 & 128 & 90 & 66 & 54 & 40 \\
\hline 6 & 5.6 & 6.1 & 1136 & 1148 & 1092 & 1005 & 945 & 718 & 470 & 241 \\
\hline 7 & 5.4 & 6.0 & 689 & 573 & 555 & 514 & 453 & 402 & 311 & 179 \\
\hline
\end{tabular}

${ }^{*} \mathrm{~V}_{\mathrm{NB}, \mathrm{GKP}}=$ volume of healthy brain receiving a given percentage of the prescription dose for the GKP plan created for a specific image set; $V_{\mathrm{NB}, \mathrm{VMAS}}=$ volume of healthy brain receiving a given percentage of the prescription dose for the VMAS plan created for a specific image set.

and increasing target dispersion exacerbate this effect. Conversely, reducing the MLC thickness and increasing the number of arcs, couch angles, and the length of each arc may allow for the creation of slightly more conformal low-dose distributions. ${ }^{12}$ However, because only 1 isocenter is used, there is inherently more beam overlap than in the GKP. Again, this increases the volume of the lower isodose levels compared with GKP. The placement of additional VMAS isocenters greatly increases setup time, which would limit its usefulness for the treatment of multiple metastases.
The clinical significance of additional radiation spillage to the normal brain requires further research. Studies have shown that certain structures within the brain, such as the hippocampus, may be exquisitely sensitive to radiation..$^{5}$ Because of the role of the hippocampus in the formation of new memories, reducing the radiation dose to this brain region could result in improved neurocognitive and functional outcomes. The innate property of GKP to minimize the amount of healthy brain tissues receiving any given dose provides a clear advantage for sparing these structures during the treatment of multiple
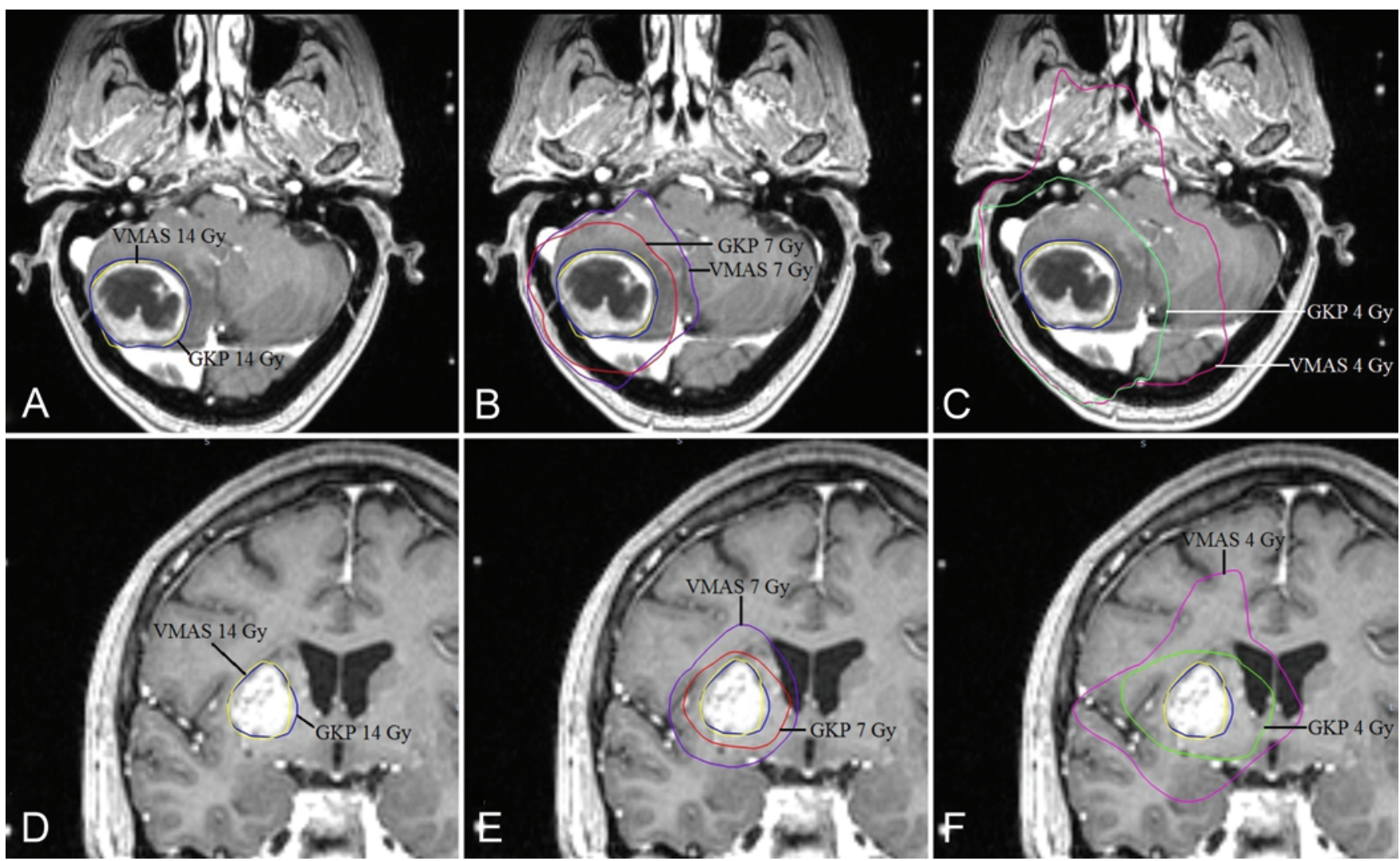

FIG. 2. Multimodality overlay of different isodose levels for the same image set. A-C: Transverse view of a tumor target. D-F: Coronal view of a different target. Gamma Knife Perfexion 14-, 7-, and 4-Gy lines are shown in blue, red, and green, respectively. Volumetric modulated arc radiosurgery 14-, 7-, and 4-Gy lines are shown in yellow, purple, and magenta, respectively. 


\section{Gamma Knife Perfexion versus VMAS}

TABLE 7: Radiosurgery quality metrics calculated for each plan

\begin{tabular}{|c|c|c|c|c|c|c|c|c|c|}
\hline \multirow[b]{2}{*}{ Metric } & \multirow[b]{2}{*}{ Ideal Value } & \multirow[b]{2}{*}{ Modality } & \multicolumn{7}{|c|}{ Calculated Value Ordered by Image Set } \\
\hline & & & 1 & 2 & 3 & 4 & 5 & 6 & 7 \\
\hline PITV & 1 & GKP & 1.75 & 1.45 & 1.57 & 1.95 & 1.35 & 3.41 & 3.43 \\
\hline \multirow[t]{2}{*}{$\mathrm{Cl}$} & 1 & GKP & 0.57 & 0.70 & 0.64 & 0.51 & 0.74 & 0.29 & 0.29 \\
\hline & & VMAS & 0.56 & 0.67 & 0.59 & 0.51 & 0.71 & 0.26 & 0.26 \\
\hline \multirow[t]{2}{*}{ CGI } & highest achievable & GKP & 69.34 & 70.24 & 55.09 & 51.33 & 49.67 & 54.62 & 53.98 \\
\hline & & VMAS & 47.43 & 44.75 & -3.83 & 7.38 & 13.78 & -27.41 & -6.15 \\
\hline
\end{tabular}

metastases. In addition, as mentioned above, because of improved systemic therapy, patients are now more likely to receive multiple radiosurgery treatments over a number of months or even years.

As the total number of metastases treated increases, it is crucial to minimize the amount of normal brain irradiated during each treatment session. Failure to do so may eventually lead to cumulative brain doses to healthy tissues high enough to cause the late-term effects that radiosurgery strategies seek to avoid. Such failure may also preclude further radiosurgical treatments because of doses exceeding the radiation tolerances of sensitive normal tissues. Finally, radiosurgery has also been successfully and safely used in salvage settings after WBRT. ${ }^{7}$ In this particular clinical application, the ability to minimize low-dose spillage to eloquent regions may allow for more aggressive salvage strategies and reduce the likelihood that patients will develop clinically detectable toxicity from high cumulative doses of radiation.

\section{Comparison of Radiosurgery Plan Quality Metrics}

Quality metrics for evaluating target coverage and prescription isodose conformality in the radiosurgery plans showed little dependence on modality. This indicates only minor differences between the 2 treatment platforms in their capability to generate acceptably conformal prescription isodose lines. Furthermore, it demonstrated that traditional conformality metrics may not provide sufficient contrast when evaluating modern radiosurgical techniques. While these traditional metrics are useful for evaluating general plan quality, most modern treatment platforms are now exceedingly capable of shaping the prescription isodose line. However, metrics that include a measure of the dose fall-off clearly show GKP to be superior. As treatment of multiple metastases and the delivery of multiple radiosurgery sessions become more common, evaluation of plan quality must focus not only on prescription isodose conformality, but also on conformality at lower isodose lines, which will enable drawing true distinctions between modern radiosurgical treatment modalities.

\section{Study Limitations}

Although we attempted to conduct and report as fair and accurate a comparison as possible, treatment plan- ning studies, by nature, have inherent limitations. Because these studies depend on the quality of the treatment plans analyzed, the planner's skill with each modality, as well as subjective criteria for plan acceptability, can potentially impact the results. For this study, an experienced physicist, with 5 years' experience of planning radiosurgery on both Gamma Knife and accelerator-based platforms, prepared all treatment plans. Plans for each modality were subjected to the same target-coverage criteria; in addition, prescription isodose conformality was adjusted to be as equivalent as possible. We believe that the uniform target-coverage criteria and the equivalent values obtained for metrics involving conformality of the prescription isodose line indicate that the plans created for each modality are of similar quality and can be reasonably compared.

Another study limitation was the relatively low number of plans analyzed. While we feel that the results of this study are clear, adding additional plans for analysis could further improve the power of a future study.

Finally, we compared plans created using the specific tools available in our clinic at the time of this study. There are variations in planning and delivery platforms, especially for VMAS, that could potentially impact the magnitude of the results seen here. For example, micro MLCs have recently been made available for acceleratorbeam shaping. These MLCs range in size from 0.25 to 0.5 $\mathrm{cm}$, in contrast to the standard MLCs, used for this work, which ranged in size from 0.5 to $1.0 \mathrm{~cm}$ and are most commonly used in current clinical practice. The smaller MLCs may help to improve the prescription isodose conformality of VMAS plans. Clearly, future studies will have to take into account new treatment technologies as they become disseminated.

\section{Literature Review}

The current literature examining low-dose spillage during the treatment of multiple metastases is limited, and few reports have addressed this topic for different planning and delivery platforms. Studies by Ma and colleagues have compared GKP to a number of different treatment modalities, using a single data set and treating variable numbers of metastases. ${ }^{11,12,14}$ The modalities used include GKP, CyberKnife, and single-isocenter VMAS for delivery on Novalis and Varian TrueBeam ac- 
celerators. These accelerators were equipped with micro $(0.3-0.25 \mathrm{~cm})$ MLCs. In each study, GKP was found to deliver significantly less dose to normal brain tissues than competing modalities. Although the accelerator delivery platforms are different, these results agree with our findings and point to GKP as providing superior conformality for low isodose lines.

Thomas et al. recently reported a comparison of the Gamma Knife model 4C (GK4C) with a Varian TrueBeam with micro MLCs. ${ }^{21}$ This study examined data from 28 patients with multiple metastases previously planned for GK4C and replanned for single-isocenter VMAS. Analyzing the average values over all data sets, the authors found significantly improved conformality of the prescription isodose for VMAS. In addition, although the average values for a majority of the dose-spillage metrics examined were worse for VMAS than for GK4C, these differences were not statistically significant. They concluded that single-isocenter VMAS provides prescription isodose conformality superior to Gamma Knife, with no statistically significant increase in low-dose spillage.

While these results seem to contradict those of Ma and colleagues ${ }^{11,12,14}$ and of our own group, equipment limitations leading to differences in plan quality, especially prescription isodose conformality, likely explain this discrepancy. The GK4C, used by Thomas et al., ${ }^{21}$ is an older version of the Gamma Knife. Changing collimator sizes on the GK4C is considerably more time consuming than on the GKP. In addition, the creation of custom collimators, while common on the GKP, is difficult and rare on the GK4C. As a result, treatments for multiple metastases planned on the GK4C tend to use fewer shots and larger collimators, sacrificing conformality for ease of treatment and time savings. This is demonstrated by the significantly improved prescription isodose conformality seen by Thomas and colleagues for the VMAS platform compared with GK4C. ${ }^{21}$

Naturally, as prescription isodose conformality decreases, low-dose spillage increases. Despite the superior quality of the VMAS plans analyzed by Thomas et al., ${ }^{21} \mathrm{a}$ majority of the examined dose-spillage metrics were improved for GK4C compared with VMAS, though not by a statistically significant degree. The plans analyzed by Ma and colleagues ${ }^{11,12,14}$ and by our own group had GKP and VMAS prescription isodose conformalities that were essentially equivalent, with Ma and colleagues showing slightly improved conformality for GKP when treating $\geq$ 9 metastases. As we have reported here, in this setting of equivalent prescription isodose conformality, GKP provides significantly improved low-dose spillage compared with single-isocenter VMAS.

\section{Conclusions}

Stereotactic radiosurgery plans for the treatment of multiple metastases with the VMAS modality were shown to deliver significantly more radiation to the normal brain than plans created for the GKP treatment platform. These results were consistent for 7 different patient data sets with unique metastasis configurations and corroborated those of multiple studies using different plan- ning and delivery platforms. ${ }^{12-14}$ In addition, it has been shown that radiosurgery quality metrics including an evaluation of radiation dose gradients are better suited to discern differences between modern radiosurgery treatment modalities than metrics evaluating prescription isodose conformality alone.

As control of extracranial diseases improves, the likelihood that patients will live long enough to experience the negative cognitive effects associated with WBRT increases. Modern technology now permits the routine radiosurgical treatment of multiple metastases. Cranial disease can be successfully controlled while sparing normal brain tissues, allowing patients the potential for a better quality of life. However, as the number of metastases treated and the total number of radiosurgery treatment sessions increase, the effect of cumulative dose spillage into the healthy parts of the brain must be considered. This consideration should influence not only the choice between existing treatment platforms, but also the future direction of treatment system development. It is essential that modern radiosurgery treatment modalities minimize this dose spillage across all dose levels.

\section{Disclosure}

The authors report no conflict of interest concerning the materials or methods used in this study or the findings specified in this paper.

Author contributions to the study and manuscript preparation include the following. Conception and design: McDonald, Takacs, Jenrette, Vanek. Acquisition of data: McDonald. Analysis and interpretation of data: McDonald. Drafting the article: all authors. Critically revising the article: all authors. Reviewed submitted version of manuscript: all authors. Approved the final version of the manuscript on behalf of all authors: McDonald. Statistical analysis: McDonald. Administrative/technical/material support: McDonald, Vanek. Study supervision: McDonald, Takacs, Peng, Vanek.

\section{References}

1. Audet C, Poffenbarger BA, Chang P, Jackson PS, Lundahl RE, Ryu SI, et al: Evaluation of volumetric modulated arc therapy for cranial radiosurgery using multiple noncoplanar arcs. Med Phys 38:5863-5872, 2011

2. Bindal RK, Sawaya R, Leavens ME, Lee JJ: Surgical treatment of multiple brain metastases. J Neurosurg 79:210-216, 1993

3. Chang EL, Wefel JS, Hess KR, Allen PK, Lang FF, Kornguth DG, et al: Neurocognition in patients with brain metastases treated with radiosurgery or radiosurgery plus whole-brain irradiation: a randomised controlled trial. Lancet Oncol 10: 1037-1044, 2009

4. Clark GM, Popple RA, Young PE, Fiveash JB: Feasibility of single-isocenter volumetric modulated arc radiosurgery for treatment of multiple brain metastases. Int J Radiat Oncol Biol Phys 76:296-302, 2010

5. Gondi V, Hermann BP, Mehta MP, Tomé WA: Hippocampal dosimetry predicts neurocognitive function impairment after fractionated stereotactic radiotherapy for benign or low-grade adult brain tumors. Int J Radiat Oncol Biol Phys 83: $487-$ e493, 2012

6. Hardcastle N, Tome WA: On a single isocenter volumetric modulated arc therapy SRS planning technique for multiple brain metastases. J Radiosurg SBRT 2:1-9, 2012

7. Harris S, Chan MD, Lovato JF, Ellis TL, Tatter SB, Bourland $\mathrm{JD}$, et al: Gamma knife stereotactic radiosurgery as salvage 
therapy after failure of whole-brain radiotherapy in patients with small-cell lung cancer. Int J Radiat Oncol Biol Phys 83:e53-e59, 2012

8. Kalkanis SN, Kondziolka D, Gaspar LE, Burri SH, Asher AL, Cobbs CS, et al: The role of surgical resection in the management of newly diagnosed brain metastases: a systematic review and evidence-based clinical practice guideline. J Neurooncol 96:33-43, 2010

9. Kang J, Ford EC, Smith K, Wong J, McNutt TR: A method for optimizing LINAC treatment geometry for volumetric modulated arc therapy of multiple brain metastases. Med Phys 37:4146-4154, 2010

10. Lagerwaard FJ, Levendag PC, Nowak PJ, Eijkenboom WM, Hanssens PE, Schmitz PI: Identification of prognostic factors in patients with brain metastases: a review of 1292 patients. Int J Radiat Oncol Biol Phys 43:795-803, 1999

11. Ma L, Hossain S, Nichol A, Higby C, Ahmad S, Petti P, et al: Normal brain tissue dose following stereotactic radiosurgery (SRS) of multiple brain metastases: variation across modern SRS treatment platforms. J Radiosurg SBRT 2 Suppl 1:1819, 2013 (Abstract)

12. Ma L, Nichol A, Hossain S, Wang B, Petti P, Vellani R, et al: Variable dose interplay effects across radiosurgical apparatus in treating multiple brain metastases. Int J Comput Assist Radiol Surg [epub ahead of print], 2014

13. Ma L, Petti P, Wang B, Descovich M, Chuang C, Barani IJ, et al: Apparatus dependence of normal brain tissue dose in stereotactic radiosurgery for multiple brain metastases. Technical note. J Neurosurg 114:1580-1584, 2011

14. Ma L, Sahgal A, Wang B, Hossain S, Ahmad S, Larson D: SU-E-T-669: dose interplay effects in stereotactic radiosurgery (SRS) of multiple brain lesions. Med Phys 40:360, 2013 (Abstract)

15. Paddick I: A simple scoring ratio to index the conformity of radiosurgical treatment plans. Technical note. J Neurosurg 93 Suppl 3:219-222, 2000

16. Paddick I, Lippitz B: A simple dose gradient measurement tool to complement the conformity index. J Neurosurg 105 Suppl:194-201, 2006

17. Patchell RA, Tibbs PA, Walsh JW, Dempsey RJ, Maruyama Y, Kryscio RJ, et al: A randomized trial of surgery in the treatment of single metastases to the brain. N Engl J Med 322:494500,1990
18. Sawaya R, Wildrick D: Metastatic brain tumors: surgery perspective, in Chin LS, Regine WF (eds): Principles and Practice of Stereotactic Radiosurgery. New York: Springer, 2008, pp 193-200

19. Shaw E, Kline R, Gillin M, Souhami L, Hirschfeld A, Dinapoli R, et al: Radiation Therapy Oncology Group: radiosurgery quality assurance guidelines. Int J Radiat Oncol Biol Phys 27:1231-1239, 1993

20. Suh JH: Stereotactic radiosurgery for the management of brain metastases. N Engl J Med 362:1119-1127, 2010

21. Thomas EM, Popple RA, Wu X, Clark GM, Markert JM, Guthrie BL, et al: Comparison of plan quality and delivery time between volumetric arc therapy (RapidArc) and Gamma Knife radiosurgery for multiple cranial metastases. Neurosurgery [epub ahead of print], 2014

22. Wagner TH, Bova FJ, Friedman WA, Buatti JM, Bouchet LG, Meeks SL: A simple and reliable index for scoring rival stereotactic radiosurgery plans. Int J Radiat Oncol Biol Phys 57:1141-1149, 2003

23. Welzel G, Fleckenstein K, Schaefer J, Hermann B, Kraus-Tiefenbacher U, Mai SK, et al: Memory function before and after whole brain radiotherapy in patients with and without brain metastases. Int J Radiat Oncol Biol Phys 72:1311-1318, 2008

24. Zaninovich RS, Sanchez Gonzalez F, Domitrovic L, Ypa P, Mezzadri J: Surgical treatment of brain metastases. World Federation of Neurosurgical Societies. (http://www.wfns.org/ pages/read_the_reviews_volume/151.php?rid=78\&jtype=New) [Accessed August 14, 2014]

Manuscript submitted June 18, 2014.

Accepted July 28, 2014.

Portions of this work were presented in plenary sessions at the Annual Meeting of the American Association of Physicists in Medicine, Indianapolis, IN, August 4-8, 2013, and at the Annual Meeting of the Leksell Gamma Knife Society, New York, NY, May 11-15, 2014.

Please include this information when citing this paper: DOI: 10.3171/2014.7.GKS141358.

Address correspondence to: Daniel McDonald, M.S., Department of Radiation Oncology, Medical University of South Carolina, 169 Ashley Ave., Charleston, SC 29425. email: mcdonad@musc.edu. 